\title{
Asymptotics of individual eigenvalues of a class of large Hessenberg Toeplitz matrices
}

\author{
J. M. Bogoya, A. Böttcher and S. M. Grudsky
}

\begin{abstract}
We study the asymptotic behavior of individual eigenvalues of the $n$-by- $n$ truncations of certain infinite Hessenberg Toeplitz matrices as $n$ goes to infinity. The generating function of the Toeplitz matrices is supposed to be of the form $a(t)=t^{-1}(1-t)^{\alpha} f(t)(t \in \mathbb{T})$, where $\alpha$ is a positive real number but not an integer and $f$ is a smooth function in $H^{\infty}$. The classes of generating functions considered here and in a recent paper by Dai, Geary, and Kadanoff are overlapping, and in the overlapping cases, our results imply in particular a rigorous justification of an asymptotic formula which was conjectured by Dai, Geary, and Kadanoff on the basis of numerical computations.
\end{abstract}

MSC 2010. Primary 47B35. Secondary 15A15, 15A18, 47N50, 65F15

Keywords. Toeplitz matrix, eigenvalue, Fourier integral, asymptotic expansion

\section{Introduction and main results}

The $n \times n$ Toeplitz matrix generated by a complex-valued function $a \in L^{1}$ on the unit circle $\mathbb{T}$ is the matrix $T_{n}(a)=\left(a_{j-k}\right)_{j, k=0}^{n-1}$, where $a_{k}$ is the $k$ th Fourier coefficient of the function $a$, that is, $a_{k}=\int_{0}^{2 \pi} a\left(e^{i \theta}\right) e^{-i k \theta} d \theta / 2 \pi, k \in \mathbb{Z}$. The function $a$ is referred to as the symbol of the matrices $T_{n}(a)$.

If $a$ is real-valued, then the matrices $T_{n}(a)$ are all Hermitian, and in this case a number of results on the asymptotics of the eigenvalues of $T_{n}(a)$ is known; see, for example, [6], [7], [13], [16], [18], [20], [21], [23], [24], [26], [27], [29], [30]. We here consider genuinely complex-valued symbols, in which case the overall picture is less complete. Papers [12], [15], [19] describe the limiting behavior of the eigenvalues of $T_{n}(a)$ if $a$ is a rational function, while papers [1] and [28] are devoted to the asymptotic eigenvalue distribution in the case of non-smooth symbols. In [25] and [28], it is in particular shown that if $a \in L^{\infty}$ and the essential range $\mathcal{R}(a)$ does

We acknowledge support of this work by a grant of the DAAD and by CONACYT grant 219345 . 
not separate the plane, then the eigenvalues of $T_{n}(a)$ approximate $\mathcal{R}(a)$. Many of the results of the papers cited above can also be found in the books [5], [8], [9].

Throughout what follows we assume that $a$ is a complex-valued continuous function on $\mathbb{T}$. In that case $\mathcal{R}(a)=a(\mathbb{T})$. When the eigenvalues of $T_{n}(a)$ approach $\mathcal{R}(a)$ asymptotically in the sense that

$$
\lim _{n \rightarrow \infty} \frac{\operatorname{trace} \varphi\left(T_{n}(a)\right)}{n}=\int_{0}^{2 \pi} \varphi\left(a\left(e^{i \theta}\right)\right) \frac{d \theta}{2 \pi}
$$

for a sufficiently rich supply of test functions $\varphi$, one says that they have canonical distribution. In 1990, Widom [28] showed that if $\mathcal{R}(a)$ is a Jordan curve and $a$ is smooth on $\mathbb{T}$ minus a single point but not smooth on all of $\mathbb{T}$, then the spectrum of $T_{n}(a)$ has canonical distribution. He also raised the following intriguing conjecture, which is still an open problem:

The eigenvalues of $T_{n}(a)$ are canonically distributed except when

a extends analytically to an annulus $r<|z|<1$ or $1<|z|<R$.

Results like (1.1) or of the type that the spectrum of $T_{n}(a)$ converges to some limiting set in the Hausdorff metric do not provide us with information on the asymptotic behavior of individual eigenvalues. The asymptotic behavior of the extreme eigenvalues of Hermitian Toeplitz matrices is fairly well understood; see the references cited above. Paper [6] contains asymptotic expansions for individual inner eigenvalues of certain banded Hermitian Toeplitz matrices. The recent papers [11] and [17] concern asymptotic formulas for individual eigenvalues of Toeplitz matrices whose symbols are complex-valued and have a so-called Fisher-Hartwig singularity. These are special symbols that are smooth on $\mathbb{T}$ minus a single point but not smooth on the entire circle $\mathbb{T}$; see [8], [9].

To be more specific, Dai, Geary, and Kadanoff [11] considered symbols of the form

$$
a(t)=\left(2-t-\frac{1}{t}\right)^{\gamma}(-t)^{\beta}, \quad t \in \mathbb{T},
$$

where $0<\gamma<-\beta<1$. They conjectured that the eigenvalues $\lambda_{j}=\lambda_{j, n}$ satisfy

$$
\lambda_{j} \approx a\left(n^{(2 \gamma+1) / n} \exp \left(-\frac{2 \pi i}{n} j\right)\right), \quad j=0, \ldots, n-1,
$$

and confirmed this conjecture numerically.

Let $H^{\infty}$ be the usual Hardy space of (boundary values of) bounded analytic functions in the unit disk $\mathbb{D}$. Given $a \in C(\mathbb{T})$, we denote by wind ${ }_{\lambda}(a)$ the winding number of $a$ about a point $\lambda \in \mathbb{C} \backslash \mathcal{R}(a)$ and by $\mathcal{D}(a)$ the set of all $\lambda \in \mathbb{C}$ for

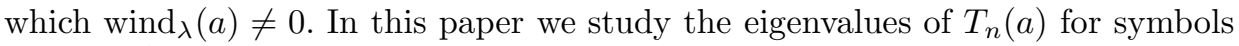
$a(t)=t^{-1} h(t)$ with the following properties:

1. $h \in H^{\infty}$ and $h_{0} \neq 0$;

2. $h(t)=(1-t)^{\alpha} f(t)$, where $\alpha \in[0, \infty) \backslash \mathbb{Z}$ and $f \in C^{\infty}(\mathbb{T})$;

3. $h$ has an analytic extension to an open neighborhood $W$ of $\mathbb{T} \backslash\{1\}$ not containing the point 1 ;

4. $\mathcal{R}(a)$ is a Jordan curve in $\mathbb{C}$ and $\operatorname{wind}_{\lambda}(a)=-1$ for each $\lambda \in \mathcal{D}(a)$. 
According to [28], in our case the spectrum of $T_{n}(a)$ has canonical distribution. Note that when $\beta=\gamma-1$ and $f \equiv 1$, our symbol coincides with the one of [11].

Let $D_{n}(a)$ denote the determinant of $T_{n}(a)$. Thus, the eigenvalues $\lambda$ of $T_{n}(a)$ are the solutions of the equation $D_{n}(a-\lambda)=0$. Our assumptions imply that $T_{n}(a)$ is a Hessenberg matrix, that is, it arises from a lower triangular matrix by adding the superdiagonal. This circumstance together with the Baxter-Schmidt formula for Toeplitz determinants allows us to express $D_{n}(a-\lambda)$ as a Fourier integral. The value of this integral mainly depends on $\lambda$ and on the singularity of $(1-t)^{\alpha}$ at the point 1 . Let $W_{0}$ be a small open neighborhood of zero in $\mathbb{C}$. We show that for every point $\lambda \in \mathcal{D}(a) \cap\left(a(W) \backslash W_{0}\right)$ there exists a unique point $t_{\lambda} \notin \overline{\mathbb{D}}$ such that $a\left(t_{\lambda}\right)=\lambda$. After exploring the contributions of $\lambda$ and the singular point 1 to the Fourier integral, we get the following asymptotic expansion for $D_{n}(a-\lambda)$.

Theorem 1.1. Let $a(t)=t^{-1} h(t)$ be a symbol with properties 1 to 4 . Then for every small open neighborhood $W_{0}$ of zero in $\mathbb{C}$ and every $\lambda \in \mathcal{D}(a) \cap\left(a(W) \backslash W_{0}\right)$,

$$
D_{n}(a-\lambda)=\left(-h_{0}\right)^{n+1}\left[\frac{1}{t_{\lambda}^{n+2} a^{\prime}\left(t_{\lambda}\right)}-\frac{f(1) \Gamma(\alpha+1) \sin (\alpha \pi)}{\pi \lambda^{2} n^{\alpha+1}}+R_{1}(n, \lambda)\right],
$$

where $R_{1}(n, \lambda)=\mathcal{O}\left(n^{-\alpha-\alpha_{0}-1}\right)$ as $n \rightarrow \infty$, uniformly in $\lambda \in a(W) \backslash W_{0}$. Here $\alpha_{0}=\min \{\alpha, 1\}$ and $h_{0}$ is the zeroth Fourier coefficient of $h$.

The first term in brackets is the contribution of $\lambda$, while the second is the contribution of the point 1 .

Here now are our main results. Let $W_{0}$ be a small open neighborhood of the origin in $\mathbb{C}$ and put $\omega_{j}:=\exp (-2 \pi i j / n)$. For each $n$ there exists integers $n_{1}$ and $n_{2}$ such that $\omega_{n_{1}}, \omega_{n-n_{2}} \in a^{-1}\left(W_{0}\right)$ but $\omega_{n_{1}+1}, \omega_{n-n_{2}-1} \notin a^{-1}\left(W_{0}\right)$. Recall that $a\left(t_{\lambda}\right)=\lambda$.

Theorem 1.2. Let $a(t)=t^{-1} h(t)$ be a symbol with properties 1 to 4. Then for every small open neighborhood $W_{0}$ of the origin in $\mathbb{C}$ and every $j$ between $n_{1}$ and $n-n_{2}$,

$$
t_{\lambda_{j}}=n^{(\alpha+1) / n} \omega_{j}\left[1+\frac{1}{n} \log \left(\frac{a^{2}\left(\omega_{j}\right)}{c_{0}(1) a^{\prime}\left(\omega_{j}\right) \omega_{j}^{2}}\right)+R_{2}(n, j)\right],
$$

where $R_{2}(n, j)=\mathcal{O}\left(n^{-\alpha_{0}-1}\right)+\mathcal{O}\left(n^{-2} \log n\right)$ as $n \rightarrow \infty$, uniformly with respect to $j$ in $\left(n_{1}, n-n_{2}\right)$. Here $\alpha_{0}=\min \{\alpha, 1\}$ and

$$
c_{0}(1)=\frac{f(1) \Gamma(\alpha+1) \sin (\alpha \pi)}{\pi} .
$$

Formula (1.4) proves conjecture (1.2) in the special case $\beta=\gamma-1$. It shows that as $n$ increases, the point $t_{\lambda_{j}}$ is close to $n^{(\alpha+1) / n} \omega_{j}$. Finally, we take the value of $a$ at the point (1.4) to obtain the following expression for $\lambda_{j}$. 
Theorem 1.3. Let $a(t)=t^{-1} h(t)$ be a symbol with properties 1 to 4 . Then for every small neighborhood $W_{0}$ of zero in $\mathbb{C}$ and every $j$ between $n_{1}$ and $n-n_{2}$,

$$
\begin{aligned}
\lambda_{j}= & a\left(\omega_{j}\right)+(\alpha+1) \omega_{j} a^{\prime}\left(\omega_{j}\right) \frac{\log n}{n} \\
& +\frac{\omega_{j} a^{\prime}\left(\omega_{j}\right)}{n} \log \left(\frac{a^{2}\left(\omega_{j}\right)}{c_{0}(1) a^{\prime}\left(\omega_{j}\right) \omega_{j}^{2}}\right)+R_{3}(n, j),
\end{aligned}
$$

where $c_{0}(1)$ is as in Theorem 1.2 and $R_{3}(n, j)=\mathcal{O}\left(n^{-\alpha_{0}-1}\right)+\mathcal{O}\left(n^{-2}(\log n)^{2}\right)$ as $n \rightarrow \infty$, uniformly with respect to $j$ in $\left(n_{1}, n-n_{2}\right)$.

In the case where $f$ is identically 1 , the previous three theorems are essentially already in [3]. The idea to use just the singularity $(1-t)^{\alpha}$ in order to study phenomena connected with eigenvalue asymptotics was also employed in [4].

We remark that we wrote down only the first few terms in our asymptotic expansions but that our method is constructive and would allow us to get as many terms as we desire. Clearly, conjecture (1.2) corresponds to the first term in our asymptotic expansion (1.4). Figure 1 illustrates Theorem 1.3. In the last section, we present another simulation graphic and error tables made with Matlab software to show that incorporating the second term of our expansion (1.4) (= third term in $(1.5))$ reduces the error to nearly one tenth.

\section{Toeplitz determinant}

Lemma 2.1. Let $a(t)=t^{-1} h(t)$ have properties 1 and 4. Then, for each $\lambda \in \mathcal{D}(a)$ and every $n \in \mathbb{N}$, and with [ ] $]_{n}$ denoting the nth Fourier coefficient,

$$
D_{n}(a-\lambda)=(-1)^{n} h_{0}^{n+1}\left[\frac{1}{h(t)-\lambda t}\right]_{n} .
$$

Proof. This can be deduced from the Baxter-Schmidt formula [2], which is also in $[5$, p. 37]. For the reader's convenience, we include a direct proof of (2.1). Obviously,

and

$$
T_{n+1}(h-\lambda t)=\left[\begin{array}{lllll|l}
h_{0} & 0 & 0 & \cdots & 0 & 0 \\
\hline h_{1}-\lambda & h_{0} & 0 & \cdots & 0 & 0 \\
h_{2} & h_{1}-\lambda & h_{0} & \cdots & 0 & 0 \\
\vdots & \vdots & \vdots & \ddots & \vdots & \vdots \\
h_{n-1} & h_{n-2} & h_{n-3} & \cdots & h_{0} & 0 \\
h_{n} & h_{n-1} & h_{n-2} & \cdots & h_{1}-\lambda & h_{0}
\end{array}\right]
$$

$$
T_{n}(a-\lambda)=\left[\begin{array}{lllll}
h_{1}-\lambda & h_{0} & 0 & \cdots & 0 \\
h_{2} & h_{1}-\lambda & h_{0} & \cdots & 0 \\
\vdots & \vdots & \vdots & \ddots & \vdots \\
h_{n-1} & h_{n-2} & h_{n-3} & \cdots & h_{0} \\
h_{n} & h_{n-1} & h_{n-2} & \cdots & h_{1}-\lambda
\end{array}\right] .
$$




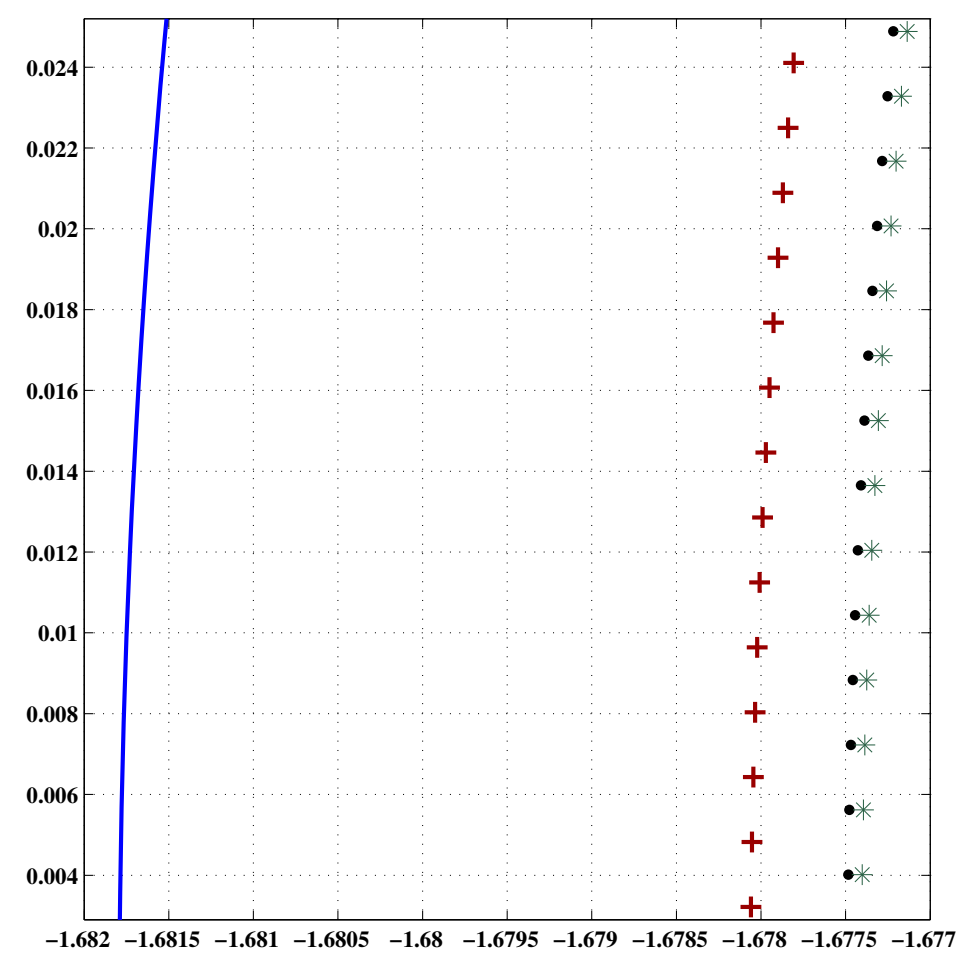

FiguRe 1. The picture shows a piece of $\mathcal{R}(a)$ for the symbol $a(t)=t^{-1}(1-t)^{3 / 4}$ (solid line) located "far" from zero. The dots are sp $T_{4096}(a)$ calculated by Matlab. The crosses and the stars are the approximations obtained by using 2 and 3 terms of (1.5), respectively.

Applying Cramer's rule to (2.2) we obtain

$$
\left[T_{n+1}^{-1}(h-\lambda t)\right]_{(n+1,1)}=(-1)^{n+2} \frac{D_{n}(a-\lambda)}{D_{n+1}(h-\lambda t)} .
$$

We claim that $h(t)-\lambda t$ is invertible in $H^{\infty}$. To see this, we must show that $h(t) \neq \lambda t$ for all $t \in \overline{\mathbb{D}}$ and each $\lambda \in \mathcal{D}(a)$. Let $\lambda$ be a point in $\mathcal{D}(a)$. For each $t \in \mathbb{T}$ we have $h(t) \neq \lambda t$ because $\lambda \notin \partial \mathcal{D}(a)=\mathcal{R}(a)$. By assumption, wind ${ }_{\lambda}(a)=-1$ and thus

$$
\begin{aligned}
-1 & =\operatorname{wind}_{0}(a-\lambda)=\operatorname{wind}_{0}\left(t^{-1} h(t)-\lambda\right)=\operatorname{wind}_{0}\left(t^{-1}(h(t)-\lambda t)\right) \\
& =\operatorname{wind}_{0}\left(t^{-1}\right)+\operatorname{wind}_{0}(h(t)-\lambda t)=-1+\operatorname{wind}_{0}(h(t)-\lambda t) .
\end{aligned}
$$


It follows that wind ${ }_{0}(h(t)-\lambda t)=0$, which means that the origin does not belong to the inside domain of the curve $\{h(t)-\lambda t: t \in \mathbb{T}\}$ (see [10, p. 204]). As $h \in H^{\infty}$, this shows that $h(t) \neq \lambda t$ for all $t \in \mathbb{D}$ and proves our claim.

If $b$ is invertible in $H^{\infty}$, then $T_{n+1}^{-1}(b)=T_{n+1}(1 / b)$. Thus, the $(n+1,1)$ entry of the matrix $T_{n+1}^{-1}(h(t)-\lambda t)$ is in fact the $n$th Fourier coefficient of $(h(t)-\lambda t)^{-1}$,

$$
\left[T_{n+1}^{-1}(h(t)-\lambda t)\right]_{(n+1,1)}=\left[\frac{1}{h(t)-\lambda t}\right]_{n} .
$$

Inserting this in $(2.3)$ we get

$$
\begin{aligned}
D_{n}(a-\lambda) & =(-1)^{n+2} D_{n+1}(h(t)-\lambda t)\left[\frac{1}{h(t)-\lambda t}\right]_{n} \\
& =(-1)^{n} h_{0}^{n+1}\left[\frac{1}{h(t)-\lambda t}\right]_{n},
\end{aligned}
$$

which completes the proof.

Expression (2.1) says that the determinant $D_{n}(a-\lambda)$ can be expressed as the Fourier integral

$$
D_{n}(a-\lambda)=(-1)^{n} h_{0}^{n+1} \int_{-\pi}^{\pi} \frac{e^{-i n \theta}}{h\left(e^{i \theta}\right)-\lambda e^{i \theta}} \frac{d \theta}{2 \pi},
$$

which is our starting point to find an asymptotic expansion for the eigenvalues of $T_{n}(a)$. There are two major contributions to this integral. The first comes from $\lambda$, when it is close to $\mathcal{R}(a)$, and the second results from the singularity at the point 1. We will analyze them in separate sections.

\section{Contribution of $\lambda$ to the asymptotic behavior of $D_{n}$}

Defining

we have

$$
b(z, \lambda):=\frac{1}{h(z)-\lambda z}
$$

$$
b_{n}(\lambda)=\int_{-\pi}^{\pi} b\left(e^{i \theta}, \lambda\right) e^{-i n \theta} \frac{d \theta}{2 \pi} .
$$

From (2.1) we conclude that

$$
D_{n}(a-\lambda)=(-1)^{n} h_{0}^{n+1} b_{n}(\lambda) .
$$

Lemma 3.1. Let $a(t)=t^{-1} h(t)$ be a symbol such that $\mathcal{R}(a)$ is a Jordan curve in $\mathbb{C}$. Let $W_{0}$ be a small open neighborhood of zero in $\mathbb{C}$. Assume that $h$ has an analytic extension to an open neighborhood $W$ of $\mathbb{T} \backslash\{1\}$ in $\mathbb{C}$ not containing the point 1 . Then, for each $\lambda \in \mathcal{D}(a) \backslash W_{0}$ sufficiently close to $\mathcal{R}(a)$, there exists a unique point $t_{\lambda}$ in $W \backslash \overline{\mathbb{D}}$ such that $a\left(t_{\lambda}\right)=\lambda$. Moreover, the point $t_{\lambda}$ is a simple pole for $b$. 
Proof. Without loss of generality, we may assume that the extension of $a$ to $W$ is bounded. As $h \in H^{\infty}$, this extension must map $W \backslash \overline{\mathbb{D}}$ to $\mathcal{D}(a) \cap a(W)$. As the range of $a$ has no loops, we have $a^{\prime}(t) \neq 0$ for all $t \in \mathbb{T}$. Consider the compact set $S:=\left\{t \in \mathbb{T}: a(t) \notin W_{0}\right\}$. For every $t \in S$, there exists an open neighborhood $V_{t}$ of $t$ in $\mathbb{C}$ with $V_{t} \subset W$ such that $a^{\prime}(t) \neq 0$ for each $t \in V_{t}$. Thus, there is an open set $U_{t}$ such that $t \in U_{t} \subset V_{t}$ and $a$ is a conformal map (and hence bijective) from $U_{t}$ to $a\left(U_{t}\right)$. As $S$ is compact, we can take a finite sub-cover from $\left\{U_{t}\right\}_{t \in S}$, say $U:=\cup_{i=1}^{M} U_{t_{i}}$. It follows that $a$ is a conformal map (and hence bijective) from $U \supset S$ to $a(U) \supset a(S)$; see Figure 2. The lemma then holds for every $\lambda \in a(U) \cap\left(\mathcal{D}(a) \backslash W_{0}\right)$. Finally, since $a^{\prime}\left(t_{\lambda}\right) \neq 0$, the point $t_{\lambda}$ must be a simple pole of $b$.

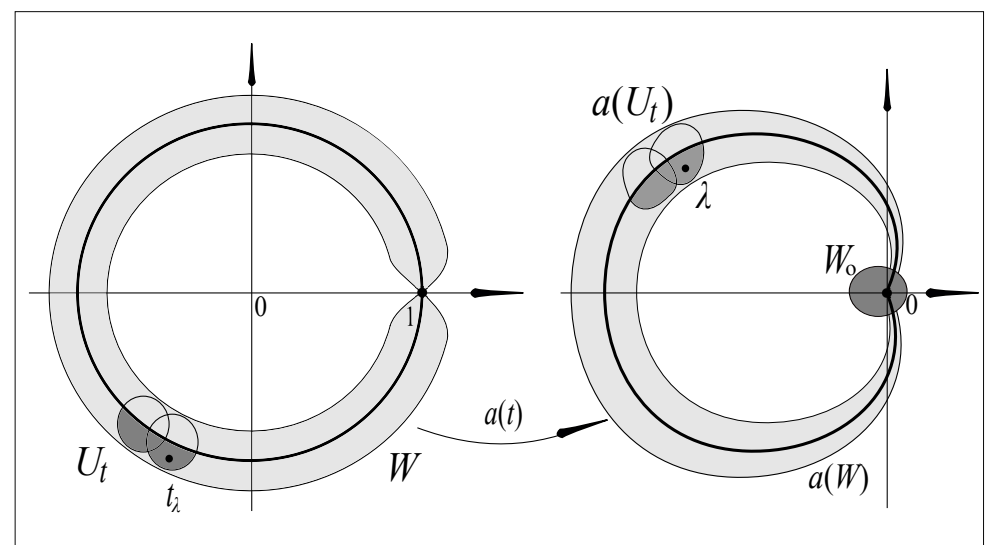

Figure 2. The map $a(t)$ over the unit circle.

Now using that $t_{\lambda}$ is a simple pole of $b$, we split $b$ as follows:

$$
b(z, \lambda)=\frac{1}{z(a(z)-\lambda)}=\frac{1}{t_{\lambda} a^{\prime}\left(t_{\lambda}\right)\left(z-t_{\lambda}\right)}+f_{0}(z, \lambda) .
$$

Here $f_{0}$ is analytic with respect to $z$ in $W$ and uniformly bounded with respect to $\lambda$ in $a(W) \backslash W_{0}$. We calculate the Fourier coefficients of the first term in (3.3) directly and integrate the second term to get

$$
b_{n}(\lambda)=\frac{-1}{t_{\lambda}^{n+2} a^{\prime}\left(t_{\lambda}\right)}+\mathcal{I},
$$

where

$$
\mathcal{I}:=\int_{-\pi}^{\pi} f_{0}\left(e^{i \theta}, \lambda\right) e^{-i n \theta} \frac{d \theta}{2 \pi}
$$

The first term in (3.4) times $(-1)^{n} h_{0}^{n+1}$ is the contribution of $t_{\lambda}$ to the asymptotic expansion of $D_{n}(a-\lambda)$; see (3.2). The function $f_{0}$ has a singularity at $z=1$ and we use this fact to expand $\mathcal{I}$ in the following section. 


\section{Contribution of 1 to the asymptotic behavior of $D_{n}$}

In this section, we will show that the value of $\mathcal{I}$ in (3.4) depends mainly on the singularity at the point 1 . Let us write $b(\theta, \lambda)$ and $f_{0}(\theta, \lambda)$ instead of $b\left(e^{i \theta}, \lambda\right)$ and $f_{0}\left(e^{i \theta}, \lambda\right)$, respectively. Let $\left\{\phi_{1}, \phi_{2}\right\}$ be a smooth partition of unity over the segment $[-\pi, \pi]$, which means that $\phi_{1}, \phi_{2} \in C^{\infty}[-\pi, \pi], \phi_{1}(\theta)+\phi_{2}(\theta)=1$ for all $\theta \in[-\pi, \pi]$, the support of $\phi_{1}$ is contained in $[-\pi,-\varepsilon] \cup[\varepsilon, \pi]$, and the support of $\phi_{2}$ is in $[-\delta, \delta]$, where $0<\varepsilon<\delta$ are small constants. By pasting segments $[-\pi, \pi]$ in both directions, we can continue $\phi_{1}$ and $\phi_{2}$ to the entire real line $\mathbb{R}$, and we will think of these two functions in that way.

Lemma 4.1. For every sufficiently small positive $\delta$, we have

$$
\mathcal{I}=\int_{-\delta}^{\delta} \phi_{2}(\theta) b(\theta, \lambda) e^{-i n \theta} \frac{d \theta}{2 \pi}+Q_{1}(n, \lambda),
$$

where $Q_{1}(n, \lambda)=\mathcal{O}\left(n^{-\infty}\right)$ as $n \rightarrow \infty$, uniformly with respect to $\lambda$ in $a(W) \backslash W_{0}$.

Proof. Using the partition of unity $\left\{\phi_{1}, \phi_{2}\right\}$, we write $\mathcal{I}=\mathcal{I}_{1}+\mathcal{I}_{2}$ where

$$
\mathcal{I}_{1}:=\int_{\varepsilon}^{2 \pi-\varepsilon} \phi_{1}(\theta) f_{0}(\theta, \lambda) e^{-i n \theta} \frac{d \theta}{2 \pi}
$$

and

$$
\mathcal{I}_{2}:=\int_{-\delta}^{\delta} \phi_{2}(\theta) f_{0}(\theta, \lambda) e^{-i n \theta} \frac{d \theta}{2 \pi} .
$$

The function $\phi_{1}(\theta) f_{0}(\theta, \lambda)$ belongs to $C^{\infty}[\varepsilon, 2 \pi-\varepsilon]$. Thus by [10, p. 22], we obtain that $\mathcal{I}_{1}=\mathcal{O}\left(n^{-\infty}\right)$ as $n \rightarrow \infty$, uniformly with respect to $\lambda$ in $a(W) \backslash W_{0}$.

Using (3.3) and writing $h(\theta)$ instead of $h\left(e^{i \theta}\right)$, we arrive at $\mathcal{I}_{2}=\mathcal{I}_{21}+\mathcal{I}_{22}$ where

$$
\mathcal{I}_{21}:=\int_{-\delta}^{\delta} \frac{\phi_{2}(\theta) e^{-i n \theta}}{h(\theta)-\lambda e^{i \theta}} \frac{d \theta}{2 \pi}
$$

and

$$
\mathcal{I}_{22}:=\frac{-1}{t_{\lambda} a^{\prime}\left(t_{\lambda}\right)} \int_{-\delta}^{\delta} \frac{\phi_{2}(\theta) e^{-i n \theta}}{e^{i \theta}-t_{\lambda}} \frac{d \theta}{2 \pi} .
$$

Once more, the function $\phi_{2}(\theta) /\left(e^{i \theta}-t_{\lambda}\right)$ belongs to $C^{\infty}[-\delta, \delta]$, we thus conclude that $\mathcal{I}_{22}=\mathcal{O}\left(n^{-\infty}\right)$ as $n \rightarrow \infty$, uniformly with respect to $\lambda$ in $a(W) \backslash W_{0}$.

Expression (4.1) says that the value of $\mathcal{I}$ basically depends on the integrand $b(\theta, \lambda) e^{-i n \theta}$ at $\theta=0$. As we can take $\delta$ as small as we desire, we can assume that $\theta$ is arbitrarily close to zero. Keeping this idea in mind, we will develop an asymptotic expansion for $b$. For future reference, we rewrite (4.1) as

$$
\mathcal{I}=\mathcal{I}_{21}+Q_{1}(n, \lambda),
$$

where $Q_{1}(n, \lambda)=\mathcal{O}\left(n^{-\infty}\right)$ as $n \rightarrow \infty$, uniformly with respect to $\lambda$ in $a(W) \backslash W_{0}$. 
Lemma 4.2. For every sufficiently small positive $\delta$,

$$
\mathcal{I}_{21}=-\sum_{s=0}^{\infty} \frac{1}{\lambda^{s+1}} \int_{-\delta}^{\delta} \frac{\phi_{2}(\theta) h^{s}(\theta) e^{-i n \theta}}{e^{i \theta(s+1)}} \frac{d \theta}{2 \pi} .
$$

Proof. From (4.2) we have

$$
\mathcal{I}_{21}=\int_{-\delta}^{\delta} \phi_{2}(\theta) b(\theta, \lambda) e^{-i n \theta} \frac{d \theta}{2 \pi}
$$

Note that

$$
b(\theta, \lambda)=\frac{1}{h(\theta)-\lambda e^{i \theta}}=\frac{-1}{\lambda e^{i \theta}} \cdot \frac{1}{1-\lambda^{-1} e^{-i \theta} h(\theta)} .
$$

As $|h(\theta)| \rightarrow 0$ when $\theta \rightarrow 0$, there exists a small positive constant $\delta$ such that

$$
\left|\lambda^{-1} e^{-i \theta} h(\theta)\right|<1
$$

for every $|\theta|<\delta$. Thus,

$$
b(\theta, \lambda)=\frac{-1}{\lambda e^{i \theta}} \sum_{s=0}^{\infty}\left(\lambda^{-1} e^{-i \theta} h(\theta)\right)^{s}=-\sum_{s=0}^{\infty} \frac{h^{s}(\theta)}{\lambda^{s+1} e^{i \theta(s+1)}}
$$

for every $|\theta|<\delta$. Inserting (4.6) in (4.5) finishes the proof.

We will use the notation

$$
\mathcal{I}_{21 s}:=\frac{1}{\lambda^{s+1}} \int_{-\delta}^{\delta} \frac{\phi_{2}(\theta) h^{s}(\theta) e^{-i n \theta}}{e^{i \theta(s+1)}} \frac{d \theta}{2 \pi} .
$$

Because $\phi_{2}(\theta) e^{-i \theta} \in C^{\infty}[-\delta, \delta]$, we have $\left.\mathcal{I}_{21 s}\right|_{s=0}=\mathcal{O}\left(n^{-\infty}\right)$ as $n \rightarrow \infty$, uniformly with respect to $\lambda$ in $a(W) \backslash W_{0}$. With the previous notation, we can rewrite (4.4) as

$$
\mathcal{I}_{21}=-\sum_{s=1}^{\infty} \mathcal{I}_{21 s}+Q_{2}(n, \lambda),
$$

where $Q_{2}(n, \lambda)=\mathcal{O}\left(n^{-\infty}\right)$ as $n \rightarrow \infty$, uniformly with respect to $\lambda$ in $a(W) \backslash W_{0}$.

Finally we will work with $\mathcal{I}_{21 s}$ and for this purpose we need the following well known result, which is, for example, in [14, p. 97].

Theorem 4.3. Let $\beta>0, \delta>0, v(\theta) \in C^{\infty}[0, \delta], v^{(s)}(\delta)=0$ for all $s \geq 0$. Then, as $n \rightarrow \infty$,

$$
\int_{0}^{\delta} \theta^{\beta-1} v(\theta) e^{i n \theta} d \theta \sim \sum_{s=0}^{\infty} \frac{a_{s}}{n^{s+\beta}}
$$

where

$$
a_{s}=\frac{v^{(s)}(0)}{s !} \Gamma(s+\beta) i^{s+\beta}
$$

and $\Gamma(z)=\int_{0}^{\infty} t^{z-1} e^{-t} d t$ is Euler's Gamma function. 
Lemma 4.4. Let $h(t)=(1-t)^{\alpha} f(t)$ with $\alpha \in \mathbb{R}_{+} \backslash \mathbb{Z}$ and $f \in C^{\infty}(\mathbb{T})$. Then

$$
\mathcal{I}_{21}=\frac{f(1) \Gamma(\alpha+1) \sin (\alpha \pi)}{\pi \lambda^{2} n^{\alpha+1}}+R_{1}(n, \lambda),
$$

where $R_{1}(n, \lambda)=\mathcal{O}\left(n^{-\alpha-\alpha_{0}-1}\right)$ with $\alpha_{0}=\min \{\alpha, 1\}$ as $n \rightarrow \infty$, uniformly with respect to $\lambda$ in $a(W) \backslash W_{0}$.

Proof. It is easy to verify that $h(\theta)=(-i \theta)^{\alpha} v(\theta) f\left(e^{i \theta}\right)$ as $\theta \rightarrow 0$, where $v(\theta)=$ $\left(i \theta^{-1}\left(1-e^{i \theta}\right)\right)^{\alpha}$, the branch of the $\alpha$ th power being the one corresponding to the argument in $(-\pi, \pi]$; note that for every sufficiently small positive $\delta$ we have $v \in C^{\infty}[-\delta, \delta]$ and $v(0)=1$. Thus,

$$
\begin{aligned}
\mathcal{I}_{21 s} & =\frac{1}{\lambda^{s+1}} \int_{-\delta}^{\delta} \phi_{2}(\theta) h^{s}(\theta) e^{-i \theta(n+s+1)} \frac{d \theta}{2 \pi} \\
& =\frac{(-i)^{\alpha s}}{\lambda^{s+1}} \int_{-\delta}^{\delta} \phi_{2}(\theta) \theta^{\alpha s} v^{s}(\theta) f^{s}\left(e^{i \theta}\right) e^{-i \theta(n+s+1)} \frac{d \theta}{2 \pi}
\end{aligned}
$$

when $\theta \rightarrow 0$. The last integral can be written as

$$
\begin{aligned}
\mathcal{I}_{21 s} & =\int_{-\delta}^{\delta} \theta^{\beta-1} w(\theta) e^{-i n \theta} d \theta \\
& =\int_{-\delta}^{0} \theta^{\beta-1} w(\theta) e^{-i n \theta} d \theta+\int_{0}^{\delta} \theta^{\beta-1} w(\theta) e^{-i n \theta} d \theta \\
& =\int_{0}^{\delta}(-\tau)^{\beta-1} w(-\tau) e^{i n \tau} d \tau+\int_{0}^{\delta} \theta^{\beta-1} w(\theta) e^{-i n \theta} d \theta \\
& =\mathcal{I}_{21 s 1}+\mathcal{I}_{21 s 2},
\end{aligned}
$$

where

$$
\begin{aligned}
\beta & :=\alpha s+1 \\
w(\theta) & :=\frac{(-i)^{\alpha s}}{2 \pi \lambda^{s+1}} \phi_{2}(\theta) v^{s}(\theta) f^{s}\left(e^{i \theta}\right) e^{-i \theta(s+1)}, \quad \theta \rightarrow 0,
\end{aligned}
$$

and

$$
\mathcal{I}_{21 s 1}:=(-1)^{\beta-1} \int_{0}^{\delta} \theta^{\beta-1} w(-\theta) e^{i n \theta} d \theta, \quad \mathcal{I}_{21 s 2}:=\int_{0}^{\delta} \theta^{\beta-1} w(\theta) e^{-i n \theta} d \theta .
$$

Note that $w( \pm \theta) \in C^{\infty}[0, \delta]$ and $w^{(s)}( \pm \delta)=0$ for all $s \in \mathbb{N}$ because $\phi_{2}(\theta) \equiv 0$ in a small neighborhood of $\pm \delta$. Applying (4.8) to $\mathcal{I}_{21 s 1}$ and $\overline{\mathcal{I}_{21 s 2}}$, we obtain

$$
\mathcal{I}_{21 s 1}=\frac{(-1)^{\alpha s} w(0) \Gamma(\alpha s+1) i^{\alpha s+1}}{n^{\alpha s+1}}+Q_{3}(s, n, \lambda)
$$

and

$$
\mathcal{I}_{21 s 2}=\frac{w(0) \Gamma(\alpha s+1) i^{-\alpha s-1}}{n^{\alpha s+1}}+Q_{4}(s, n, \lambda),
$$


where $Q_{3}(s, n, \lambda)$ and $Q_{4}(s, n, \lambda)$ are $\mathcal{O}\left(n^{-\alpha s-2}\right)$ as $n \rightarrow \infty$, uniformly with respect to $\lambda$ in $a(W) \backslash W_{0}$. Substitution of (4.11) in (4.10) yields

$$
\begin{aligned}
\mathcal{I}_{21 s} & =\frac{w(0) \Gamma(\alpha s+1)}{n^{\alpha s+1}}\left[i^{-\alpha s-1}+(-1)^{\alpha s} i^{\alpha s+1}\right]+Q_{5}(s, n, \lambda) \\
& =\frac{-c_{0}(s)}{\lambda^{s+1} n^{\alpha s+1}}+Q_{5}(s, n, \lambda)
\end{aligned}
$$

where

$$
c_{0}(s):=\frac{f^{s}(1) \Gamma(\alpha s+1) \sin (\alpha \pi s)}{\pi}
$$

and $Q_{5}(s, n, \lambda)=\mathcal{O}\left(n^{-\alpha s-2}\right)$ as $n \rightarrow \infty$, uniformly in $\lambda \in a(W) \backslash W_{0}$. From $(4.7)$ and (4.12) we obtain

$$
\mathcal{I}_{21}=\frac{c_{0}(1)}{\lambda^{2} n^{\alpha+1}}+R_{1}(n, \lambda)
$$

where $R_{1}(n, \lambda)=\mathcal{O}\left(n^{-\alpha-\alpha_{0}-1}\right)$ as $n \rightarrow \infty$, uniformly in $\lambda \in a(W) \backslash W_{0}$. Here $\alpha_{0}:=\min \{\alpha, 1\}$.

The previous calculation gives us the main asymptotic term for $\mathcal{I}_{21}$. If more terms are needed, say $m$, we must expand $\mathcal{I}_{21}$ from $\left.\mathcal{I}_{21 s}\right|_{s=1}$ to $\left.\mathcal{I}_{21 s}\right|_{s=m}$ and expand each $\mathcal{I}_{21 s}$ to $m$ terms, after which, according to the value of $\alpha$, we need to select the first $m$ principal terms.

Finally we put all the lemmas together to prove Theorem 1.1.

Proof of Theorem 1.1. The proof of this theorem is a direct application of equations (3.2), (3.4), (4.3) and (4.9).

\section{Individual eigenvalues}

In order to find the eigenvalues of the matrices $T_{n}(a)$, we need to solve the equations $D_{n}(a-\lambda)=0$. We start this section by locating the zeros of $D_{n}(a-\lambda)$.

Let $W_{0}$ be a small open neighborhood of zero in $\mathbb{C}$ and $\omega_{j}:=\exp (-2 \pi i j / n)$. For each $n$ there exists integers $n_{1}$ and $n_{2}$ such that $\omega_{n_{1}}, \omega_{n-n_{2}} \in a^{-1}\left(W_{0}\right)$ but $\omega_{n_{1}+1}, \omega_{n-n_{2}-1} \notin a^{-1}\left(W_{0}\right)$. Recall that $\lambda=a\left(t_{\lambda}\right)$. Take an integer $j$ satisfying $n_{1}<j<n-n_{2}$. Using the relations

$$
\frac{1}{t_{\lambda}^{2} a^{\prime}\left(t_{\lambda}\right)}=\frac{1}{\omega_{j}^{2} a^{\prime}\left(\omega_{j}\right)}+\mathcal{O}\left(\left|t_{\lambda}-\omega_{j}\right|\right)
$$

and

$$
\frac{1}{a^{2}\left(t_{\lambda}\right)}=\frac{1}{a^{2}\left(\omega_{j}\right)}+\mathcal{O}\left(\left|t_{\lambda}-\omega_{j}\right|\right),
$$


where $t_{\lambda}$ belongs to a small neighborhood of $\omega_{j}$, we see that the determinant $D_{n}(a-\lambda)$ in $(1.3)$ equals

$$
\begin{aligned}
& \left(-h_{0}\right)^{n+1}\left[T_{1}-T_{2}+\frac{1}{t_{\lambda}^{n}} \mathcal{O}\left(\left|t_{\lambda}-\omega_{j}\right|\right)+\frac{1}{n^{\alpha+1}} \mathcal{O}\left(\left|t_{\lambda}-\omega_{j}\right|\right)+Q_{6}\left(n, t_{\lambda}\right)\right] \\
& =\left(-h_{0}\right)^{n+1}\left[T_{1}-T_{2}+\mathcal{O}\left(\left|\frac{t_{\lambda}-\omega_{j}}{t_{\lambda}^{n}}\right|\right)+\mathcal{O}\left(\frac{\left|t_{\lambda}-\omega_{j}\right|}{n^{\alpha+1}}\right)+Q_{6}\left(n, t_{\lambda}\right)\right],
\end{aligned}
$$

where $Q_{6}\left(n, t_{\lambda}\right)=\mathcal{O}\left(n^{-\alpha-\alpha_{0}-1}\right)$ as $n \rightarrow \infty$, uniformly with respect to $t_{\lambda}$ in $W \backslash a^{-1}\left(W_{0}\right)$, and where $t_{\lambda}$ belongs to a small neighborhood of $\omega_{j}$. Here

$$
T_{1}:=\frac{1}{t_{\lambda}^{n} \omega_{j}^{2} a^{\prime}\left(\omega_{j}\right)}, \quad T_{2}:=\frac{c_{0}(1)}{a^{2}\left(\omega_{j}\right) n^{\alpha+1}},
$$

and $\alpha_{0}:=\min \{\alpha, 1\}$. Recall $c_{0}(1)$ from (4.13). Expression (5.1) makes sense only when $t_{\lambda}$ is sufficiently "close" to $\omega_{j}$ and thus, it is necessary to know whether there exists a zero of $D_{n}(a-\lambda)$ "close" to $\omega_{j}$. Let

$$
t_{\lambda}=(1+\rho) \exp (i \theta) \text {. }
$$

It is easy to verify that $T_{1}-T_{2}=0$ if and only if

$$
\rho=\left(\frac{\left|a\left(\omega_{j}\right)\right|^{2} n^{\alpha+1}}{\left|c_{0}(1) a^{\prime}\left(\omega_{j}\right)\right|}\right)^{1 / n}-1
$$

and

$$
\theta=\theta_{j}=\frac{1}{n} \arg \left[\frac{a^{2}\left(\omega_{j}\right)}{c_{0}(1) \omega_{j}^{2} a^{\prime}\left(\omega_{j}\right)}\right]-\frac{2 \pi}{n}
$$

for some $j \in\{0, \ldots, n-1\}$. When $n$ tends to infinity, (5.2) shows that $\rho$ remains positive and $\rho \rightarrow 0$. The function $T_{1}-T_{2}$ has $n$ zeros with respect to $\lambda \in \mathcal{D}(a)$ given by

$$
a\left((1+\rho) e^{i \theta_{0}}\right), \quad \ldots, \quad a\left((1+\rho) e^{i \theta_{n-1}}\right) .
$$

As Lemma 3.1 establishes a 1-1 correspondence between $\lambda$ and $t_{\lambda}$, the function $D_{n}(a-\lambda)$ is analytic with respect to $\lambda$ in $a(W) \backslash W_{0}$, that is, analytic with respect to $t_{\lambda}$ in $W \backslash a^{-1}\left(W_{0}\right)$. We can therefore suppose that $T_{1}-T_{2}$ has $n$ zeros with respect to $t_{\lambda}$ in the exterior of $\overline{\mathbb{D}}$ given by

$$
t_{0}:=(1+\rho) e^{i \theta_{0}}, \quad \ldots, \quad t_{n-1}:=(1+\rho) e^{i \theta_{n-1}} .
$$

We take the function "arg" in the interval $(-\pi, \pi]$. Thus, $t_{j}=(1+\rho) e^{i \theta_{j}}$ is the nearest zero to $\omega_{j}$. Consider the neighborhood $E_{j}$ of $t_{j}$ sketched in Figure 3.

The boundary of $E_{j}$ is $\Gamma:=\Gamma_{1} \cup \Gamma_{2} \cup \Gamma_{3} \cup \Gamma_{4}$. We have chosen radial segments $\Gamma_{2}$ and $\Gamma_{4}$ so that their length is $1 / n^{\epsilon}$ with $\epsilon \in\left(0, \alpha_{0}\right)$ and all the points in $\Gamma_{2}$ have the common argument $\left(\theta_{j+1}+\theta_{j}\right) / 2$, while all the points in $\Gamma_{4}$ have the common argument $\left(\theta_{j-1}+\theta_{j}\right) / 2$. As we can see in Figure 3 , these points run from the unit circle $\mathbb{T}$ to $\left(1+1 / n^{\epsilon}\right) \mathbb{T}$. Note also that $\Gamma_{1} \subset\left(1+1 / n^{\epsilon}\right) \mathbb{T}$ and $\Gamma_{3} \subset \mathbb{T}$. 


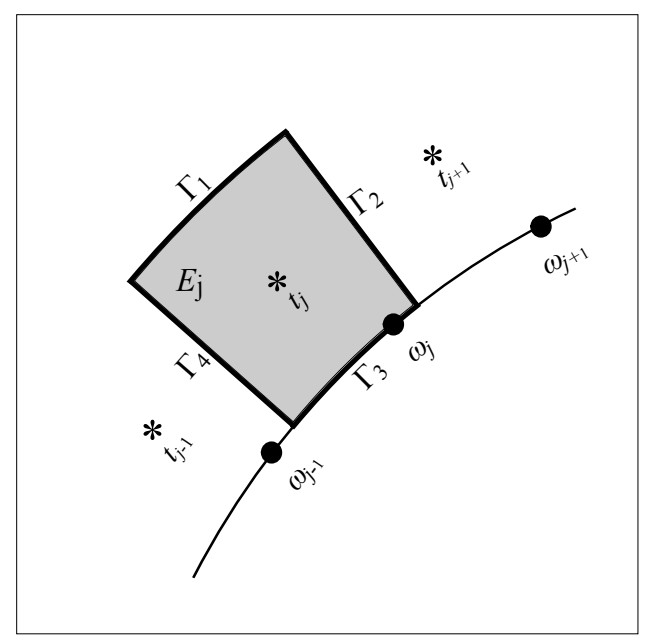

FiguRE 3 . The neighborhood $E_{j}$ of $t_{j}$ in the complex plane.

Theorem 5.1. Suppose $a(t)=t^{-1} h(t)$ is a symbol with properties 1 to 4. Let $\epsilon \in\left(0, \alpha_{0}\right)$ be a constant. Then there exists a family of sets $\left\{E_{j}\right\}_{j=n_{1}+1}^{n-n_{2}-1}$ in $\mathbb{C}$ such that

1. $\left\{E_{j}\right\}_{j=n_{1}+1}^{n-n_{2}-1}$ is a family of pairwise disjoint open sets,

2. $\operatorname{diam}\left(E_{j}\right) \leq \frac{2}{n^{\epsilon}}$,

3. $\omega_{j} \in \partial E_{j}$,

4. $D_{n}\left(a-a\left(t_{\lambda}\right)\right)=D_{n}(a-\lambda)$ has exactly one zero in each $E_{j}$.

Here $\alpha_{0}:=\min \{\alpha, 1\}$ and $\operatorname{diam}\left(E_{j}\right):=\sup \left\{\left|z_{1}-z_{2}\right|: z_{1}, z_{2} \in E_{j}\right\}$.

Proof. Assertions 1, 2, and 3 can be deduced from the above construction. We prove assertion 4 by studying the behavior of $\left|D_{n}(a-\lambda)\right|$ in dependence on $t_{\lambda} \in \Gamma$. For $t_{\lambda} \in \Gamma_{1}$ we have, as $n \rightarrow \infty$,

$$
\begin{aligned}
& \left|T_{1}\right|_{\Gamma_{1}}=\frac{1}{\left|a^{\prime}\left(\omega_{j}\right)\right|} \cdot\left(1+\frac{1}{n^{\epsilon}}\right)^{-n}=\frac{\exp \left(-n^{1-\epsilon}\right)}{\left|a^{\prime}\left(\omega_{j}\right)\right|}+\mathcal{O}\left(\frac{\exp \left(-n^{1-\epsilon}\right)}{n^{2 \epsilon-1}}\right) \\
& \left|T_{2}\right|_{\Gamma_{1}}=\frac{1}{n^{\alpha+1}} \cdot\left|\frac{c_{0}(1)}{a^{2}\left(\omega_{j}\right)}\right| \\
& \left|\mathcal{O}\left(\left|\frac{t_{\lambda}-\omega_{j}}{t_{\lambda}^{n}}\right|\right)\right|_{\Gamma_{1}}=\mathcal{O}\left(\frac{\exp \left(-n^{1-\epsilon}\right)}{n^{\epsilon}}\right) \\
& \left|\mathcal{O}\left(\frac{\left|t_{\lambda}-\omega_{j}\right|}{n^{\alpha+1}}\right)\right|_{\Gamma_{1}}=\mathcal{O}\left(\frac{1}{n^{\alpha+\epsilon+1}}\right)
\end{aligned}
$$

and

$$
\left|Q_{6}\left(n, t_{\lambda}\right)\right|_{\Gamma_{1}}=\mathcal{O}\left(\frac{1}{n^{\alpha+\alpha_{0}+1}}\right) .
$$


When $n$ goes to infinity, the absolute value of $T_{2}$ decreases at polynomial speed over $\Gamma_{1}$, while the absolute values of the remaining terms in (5.1) are smaller over $\Gamma_{1}$. Thus,

$$
\left|\frac{D_{n}(a-\lambda)}{h_{0}^{n+1}}\right|_{\Gamma_{1}}=\frac{1}{n^{\alpha+1}} \cdot\left|\frac{c_{0}(1)}{a^{2}\left(\omega_{j}\right)}\right|+\mathcal{O}\left(\frac{1}{n^{\alpha+\epsilon+1}}\right) \text { as } n \rightarrow \infty .
$$

For $t_{\lambda} \in \Gamma_{3}$ we get, as $n \rightarrow \infty$,

$$
\begin{aligned}
& \left|T_{1}\right|_{\Gamma_{3}}=\frac{1}{\left|a^{\prime}\left(\omega_{j}\right)\right|}, \quad\left|T_{2}\right|_{\Gamma_{3}}=\frac{1}{n^{\alpha+1}} \cdot\left|\frac{c_{0}(1)}{a^{2}\left(\omega_{j}\right)}\right|, \\
& \left|\mathcal{O}\left(\left|\frac{t_{\lambda}-\omega_{j}}{t_{\lambda}^{n}}\right|\right)\right|_{\Gamma_{3}}=\mathcal{O}\left(\frac{1}{n}\right), \\
& \left|\mathcal{O}\left(\frac{\left|t_{\lambda}-\omega_{j}\right|}{n^{\alpha+1}}\right)\right|_{\Gamma_{3}}=\mathcal{O}\left(\frac{1}{n^{\alpha+2}}\right),
\end{aligned}
$$

and

$$
\left|Q_{6}\left(n, t_{\lambda}\right)\right|_{\Gamma_{3}}=\mathcal{O}\left(\frac{1}{n^{\alpha+\alpha_{0}+1}}\right) .
$$

When $n$ goes to infinity, the modulus of $T_{1}$ remains constant over $\Gamma_{3}$, while the moduli of the remaining terms in (5.1) are smaller there. Consequently,

$$
\left|\frac{D_{n}(a-\lambda)}{h_{0}^{n+1}}\right|_{\Gamma_{3}}=\frac{1}{\left|a^{\prime}\left(\omega_{j}\right)\right|}+\mathcal{O}\left(\frac{1}{n}\right) \text { as } n \rightarrow \infty .
$$

As for the radial segments $\Gamma_{2}$ and $\Gamma_{4}$, we start by showing that $T_{1}$ and $-T_{2}$ have the same argument there. Since $t_{j}$ is a zero of $T_{1}-T_{2}$, we deduce that

$$
\arg \left[\frac{1}{t_{j_{0}}^{n} \omega_{j}^{2} a^{\prime}\left(\omega_{j}\right)}\right]=\arg \left[\frac{c_{0}(1)}{a^{2}\left(\omega_{j}\right) n^{\alpha+1}}\right]
$$

and thus

$$
-n \theta_{j}+\arg \left[\frac{1}{\omega_{j}^{2} a^{\prime}\left(\omega_{j}\right)}\right]=\arg \left[\frac{c_{0}(1)}{a^{2}\left(\omega_{j}\right)}\right] .
$$

For $t_{\lambda} \in \Gamma_{2}$ we have

$$
\begin{aligned}
\arg \left(T_{1}\right) & =\arg \left[\frac{1}{t_{\lambda}^{n} \omega_{j}^{2} a^{\prime}\left(\omega_{j}\right)}\right] \\
& =-\frac{n}{2}\left(\theta_{j-1}+\theta_{j}\right)+\arg \left[\frac{1}{\omega_{j}^{2} a^{\prime}\left(\omega_{j}\right)}\right] \\
& =\frac{n}{2}\left(\theta_{j}-\theta_{j-1}\right)+\arg \left[\frac{c_{0}(1)}{a^{2}\left(\omega_{j}\right)}\right] \\
& =\pi+\arg \left[\frac{c_{0}(1)}{a^{2}\left(\omega_{j}\right)}\right] \\
& =\arg \left(-T_{2}\right) .
\end{aligned}
$$


Here, the third line is due to (5.3). In addition, as $n \rightarrow \infty$,

$$
\left|\mathcal{O}\left(\left|\frac{t_{\lambda}-\omega_{j}}{t_{\lambda}^{n}}\right|\right)\right|_{\Gamma_{2}}=\mathcal{O}\left(\frac{1}{n^{\epsilon}\left|t_{\lambda}\right|^{n}}\right), \quad\left|\mathcal{O}\left(\frac{\left|t_{\lambda}-\omega_{j}\right|}{n^{\alpha+1}}\right)\right|_{\Gamma_{2}}=\mathcal{O}\left(\frac{1}{n^{\alpha+\epsilon+1}}\right),
$$

and

Furthermore,

$$
\left|Q_{6}\left(n, t_{\lambda}\right)\right|_{\Gamma_{2}}=\mathcal{O}\left(\frac{1}{n^{\alpha+\alpha_{0}+1}}\right)
$$

$$
\begin{aligned}
\left|\frac{D_{n}(a-\lambda)}{h_{0}^{n+1}}\right|_{\Gamma_{2}}= & \frac{1}{\left|t_{\lambda}^{n} a^{\prime}\left(\omega_{j}\right)\right|}+\mathcal{O}\left(\frac{1}{n^{\epsilon}\left|t_{\lambda}\right|^{n}}\right) \\
& +\frac{1}{n^{\alpha+1}} \cdot\left|\frac{c_{0}(1)}{a^{2}\left(\omega_{j}\right)}\right|+\mathcal{O}\left(\frac{1}{n^{\alpha+\epsilon+1}}\right)
\end{aligned}
$$

over $\Gamma_{2}$ when $n \rightarrow \infty$. The situation is similar for the segment $\Gamma_{4}$.

From the previous analysis of $\left|D_{n}(a-\lambda)\right|$ over $\Gamma$ we infer that for every sufficiently large $n$ we have

and

$$
\left|T_{1}-T_{2}\right|_{\Gamma} \geq \frac{1}{2 n^{\alpha+1}}\left|\frac{c_{0}(1)}{a^{2}\left(\omega_{j}\right)}\right|
$$

$$
\left|\mathcal{O}\left(\left|\frac{t_{\lambda}-\omega_{j}}{t_{\lambda}^{n}}\right|\right)+\mathcal{O}\left(\frac{\left|t_{\lambda}-\omega_{j}\right|}{n^{\alpha+1}}\right)+Q_{6}\left(n, t_{\lambda}\right)\right|_{\Gamma} \leq \mathcal{O}\left(\frac{1}{n^{\alpha+\epsilon+1}}\right) .
$$

Hence by Rouché's theorem, $D_{n}(a-\lambda) /\left(-h_{0}\right)^{n+1}$ and $T_{1}-T_{2}$ have the same number of zeros in $E_{j}$, that is, a unique zero.

As a consequence of Theorem 5.1 , we can iterate the variable $t_{\lambda}$ in the equation $D_{n}(a-\lambda)=0$, where $D_{n}(a-\lambda)$ is given by (1.3). In this fashion we find the unique eigenvalue of $T_{n}(a)$ which is located "close" to each $\omega_{j}$. We thus rewrite the equation $D_{n}(a-\lambda)=0$ in a small neighborhood of $\omega_{j}$ as

$$
t_{\lambda_{j}}=n^{(\alpha+1) / n} \omega_{j}\left[\frac{a^{2}\left(t_{\lambda_{j}}\right)}{c_{0}(1) a^{\prime}\left(t_{\lambda_{j}}\right) t_{\lambda_{j}}^{2}}\right]^{\frac{1}{n}} \cdot\left[1+Q_{7}(n, j)\right]^{-\frac{1}{n}}
$$

recall $c_{0}(1)$ from (4.13). Here the function $z^{1 / n}$ takes its principal branch, specified by the argument in $(-\pi, \pi]$. Also notice that $Q_{7}(n, j)=\mathcal{O}\left(n^{-\alpha_{0}}\right)$ as $n \rightarrow \infty$, uniformly in $j \in\left(n_{1}, n-n_{2}\right)$, with $n_{1}, n_{2}$ as in Theorem 5.1.

Proof of Theorem 1.2. Equation (5.4) is an implicit expression for $t_{\lambda_{j}}$. We manipulate it to obtain two asymptotic terms for $t_{\lambda_{j}}$. Remember that $\lambda$ belongs to $\mathcal{D}(a) \backslash W_{0}$; see Figure 2 . We can choose $W$ so thin that $\lambda_{j}=a\left(t_{\lambda_{j}}\right), a^{\prime}\left(t_{\lambda_{j}}\right)$, and $t_{\lambda_{j}}$ are bounded and not too close to zero. After expanding and multiplying the terms in brackets in (5.4), we obtain

$$
t_{\lambda_{j}}=n^{(\alpha+1) / n} \omega_{j}\left[1+\frac{1}{n} \log \left(\frac{a^{2}\left(t_{\lambda_{j}}\right)}{c_{0}(1) a^{\prime}\left(t_{\lambda_{j}}\right) t_{\lambda_{j}}^{2}}\right)+Q_{8}(n, j)\right],
$$


where $Q_{8}(n, j)=\mathcal{O}\left(n^{-\alpha_{0}-1}\right)$ as $n \rightarrow \infty$, uniformly with respect to $j$ in $\left(n_{1}, n-n_{2}\right)$. Our first approximation for $t_{\lambda_{j}}$ is

$$
t_{\lambda_{j}}=n^{(\alpha+1) / n} \omega_{j}\left[1+Q_{9}(n, j)\right]
$$

where $Q_{9}(n, j)=\mathcal{O}\left(n^{-1}\right)$ as $n \rightarrow \infty$, uniformly in $j$ from $\left(n_{1}, n-n_{2}\right)$. Replacing $t_{\lambda_{j}}$ by this approximation in (5.5) shows that $t_{\lambda_{j}}$ equals $n^{(\alpha+1) / n} \omega_{j}$ times

$$
1+\frac{1}{n} \log \left(\frac{a^{2}\left(n^{(\alpha+1) / n} \omega_{j}\left[1+Q_{9}(n, j)\right]\right)}{c_{0}(1) a^{\prime}\left(n^{(\alpha+1) / n} \omega_{j}\left[1+Q_{9}(n, j)\right]\right)\left(n^{(\alpha+1) / n} \omega_{j}\left[1+Q_{9}(n, j)\right]\right)^{2}}\right),
$$

plus $Q_{10}(n, j)$, where $Q_{10}(n, j)=\mathcal{O}\left(n^{-\alpha_{0}-1}\right)$ as $n \rightarrow \infty$, uniformly with respect to $j$ in $\left(n_{1}, n-n_{2}\right)$. Now we use the analyticity of $a$ and $a^{\prime}$ in $W$ to obtain that $t_{\lambda_{j}}$ is $n^{(\alpha+1) / n} \omega_{j}$ times

$$
1+\frac{1}{n} \log \left(\frac{a^{2}\left(n^{(\alpha+1) / n} \omega_{j}\right)}{c_{0}(1) a^{\prime}\left(n^{(\alpha+1) / n} \omega_{j}\right)\left(n^{(\alpha+1) / n} \omega_{j}\right)^{2}}\right)+Q_{11}(n, j),
$$

where $Q_{11}(n, j)=\mathcal{O}\left(n^{-\alpha_{0}-1}\right)$ as $n \rightarrow \infty$, uniformly in $j \in\left(n_{1}, n-n_{2}\right)$. Taking into account that

$$
\frac{a^{2}\left(n^{(\alpha+1) / n} \omega_{j}\right)}{c_{0}(1) a^{\prime}\left(n^{(\alpha+1) / n} \omega_{j}\right)\left(n^{(\alpha+1) / n} \omega_{j}\right)^{2}}=\frac{a^{2}\left(\omega_{j}\right)}{c_{0}(1) a^{\prime}\left(\omega_{j}\right) \omega_{j}^{2}}+\mathcal{O}\left(\frac{\log n}{n}\right) \text { as } n \rightarrow \infty
$$

we can simplify the expression for $t_{\lambda_{j}}$ to

$$
t_{\lambda_{j}}=n^{(\alpha+1) / n} \omega_{j}\left[1+\frac{1}{n} \log \left(\frac{a^{2}\left(\omega_{j}\right)}{c_{0}(1) a^{\prime}\left(\omega_{j}\right) \omega_{j}^{2}}\right)+R_{2}(n, j)\right]
$$

where $R_{2}(n, j)=\mathcal{O}\left(n^{-\alpha_{0}-1}\right)+\mathcal{O}\left(n^{-2} \log n\right)$ as $n \rightarrow \infty$, uniformly with respect to $j$ in $\left(n_{1}, n-n_{2}\right)$.

Proof of Theorem 1.3. Note that

$$
n^{(\alpha+1) / n}=\exp \left[(\alpha+1) \frac{\log n}{n}\right]=1+(\alpha+1) \frac{\log n}{n}+\mathcal{O}\left(\frac{\log n}{n}\right)^{2} \text { as } n \rightarrow \infty .
$$

Inserting (5.6) in (1.4) we obtain

$$
t_{\lambda_{j}}=\omega_{j}\left[1+(\alpha+1) \frac{\log n}{n}+\frac{1}{n} \log \left(\frac{a^{2}\left(\omega_{j}\right)}{c_{0}(1) a^{\prime}\left(\omega_{j}\right) \omega_{j}^{2}}\right)+Q_{12}(n, j)\right],
$$

where $Q_{12}(n, j)=\mathcal{O}\left(n^{-\alpha_{0}-1}\right)$ as $n \rightarrow \infty$, uniformly in $j \in\left(n_{1}, n-n_{2}\right)$. Applying the symbol $a$ to (5.7), we see that, as $n \rightarrow \infty$,

$$
\begin{aligned}
\lambda_{j}= & a\left(\omega_{j}\right)+(\alpha+1) \omega_{j} a^{\prime}\left(\omega_{j}\right) \frac{\log n}{n}+\frac{\omega_{j} a^{\prime}\left(\omega_{j}\right)}{n} \log \left(\frac{a^{2}\left(\omega_{j}\right)}{c_{0}(1) a^{\prime}\left(\omega_{j}\right) \omega_{j}^{2}}\right) \\
& +a^{\prime}\left(\omega_{j}\right) Q_{12}(n, j)+\mathcal{O}\left(\frac{\log n}{n}\right)^{2} .
\end{aligned}
$$




\section{An example}

The symbol studied by Dai, Geary, and Kadanoff [11] is

$$
a(t)=\left(2-t-\frac{1}{t}\right)^{\gamma}(-t)^{\beta}=(-1)^{3 \gamma+\beta} t^{\beta-\gamma}(1-t)^{2 \gamma}
$$

where $0<\gamma<-\beta<1$. In the case $\beta=\gamma-1$, this function $a$ becomes our symbol with $h(t)=(-1)^{4 \gamma-1}(1-t)^{2 \gamma}$. We omit the constant $(-1)^{4 \gamma-1}$, because it is just a rotation. The conjecture of [11] is that $t_{\lambda_{j}} \sim n^{(2 \gamma+1) / n} \exp (-2 \pi i j / n)$. Expansions (1.4) and (1.5) prove this result, giving us an error bound and a mathematical justification.

Our results are valid outside a small open neighborhood $W_{0}$ of the origin. Let $W_{0}=B_{1 / 5}(0)$ be the disk of radius $1 / 5$ centered at zero. Table 1 shows the data of numerical computations. It reveals that the maximum error of (1.4) with one term is reduced by nearly 10 times when considering the second term; see also Figure 1.

\begin{tabular}{|l|r|r|r|r|r|}
\hline \multicolumn{1}{|c|}{$n$} & 256 & 512 & 1024 & 2048 & 4096 \\
\hline \hline (1.4) with one term & 15.041 & 7.698 & 3.900 & 1.964 & 0.986 \\
(1.4) with two terms & 1.890 & 0.823 & 0.394 & 0.197 & 0.099 \\
(1.5) with one term & 49.374 & 27.373 & 14.983 & 8.126 & 4.376 \\
(1.5) with two terms & 14.655 & 7.574 & 3.861 & 1.952 & 0.983 \\
(1.5) with three terms & 2.633 & 1.052 & 0.479 & 0.226 & 0.109 \\
\hline
\end{tabular}

TABLE 1. The table shows the maximum error $\times 10^{3}$, obtained with our different formulas for the eigenvalues of $T_{n}\left(t^{-1}(1-t)^{3 / 4}\right)$ for different values of $n$. The data was obtained by comparison with the solutions given by Matlab, taking into account only the eigenvalues with absolute value greater than or equal to $1 / 5$.

We also performed calculations with our expansions inside $W_{0}=B_{1 / 5}(0)$, and although the error is nearly 8 times the one of outside, the approximation is still valid there because the distance between two consecutive eigenvalues is bigger than the one between an eigenvalue and the respective approximation given by (1.4) with two terms; compare Tables 1 and 2 and see Figure 4. Clearly, to describe the asymptotic behavior of the eigenvalues of $T_{n}(a)$ completely with mathematical rigor, we need an expression valid inside $W_{0}$. We hope to do this in future work.

We remark that if $\lambda$ is an eigenvalue of $T_{n}(a)$ and $b_{j}(\lambda)$ is defined by (3.1), then $\left(b_{j}(\lambda)\right)_{j=0}^{n-1}$ is an eigenvector for $\lambda$ provided $b_{n-1}(\lambda) \neq 0$. In a forthcoming paper we will employ this observation to study the asymptotics of the eigenvectors.

We finally want to emphasize that the results of this paper can be easily translated to the case where the symbol is $\overline{a(t)}=t\left(1-t^{-1}\right)^{\alpha} \overline{f(t)}$. 


\begin{tabular}{|l|r|r|r|r|r|}
\hline \multicolumn{1}{|c|}{$n$} & 256 & 512 & 1024 & 2048 & 4096 \\
\hline \hline (1.4) with one term & 29.719 & 22.395 & 16.050 & 11.123 & 7.528 \\
(1.4) with two terms & 6.229 & 3.756 & 2.247 & 1.340 & 0.797 \\
(1.5) with one term & 37.328 & 22.423 & 13.406 & 7.993 & 4.760 \\
(1.5) with two terms & 35.062 & 25.994 & 18.573 & 12.914 & 8.796 \\
(1.5) with three terms & 3.178 & 1.867 & 1.107 & 0.658 & 0.391 \\
\hline
\end{tabular}

TABLE 2. The same as in Table 1 , only now considering eigenvalues with absolute value less than $1 / 5$.

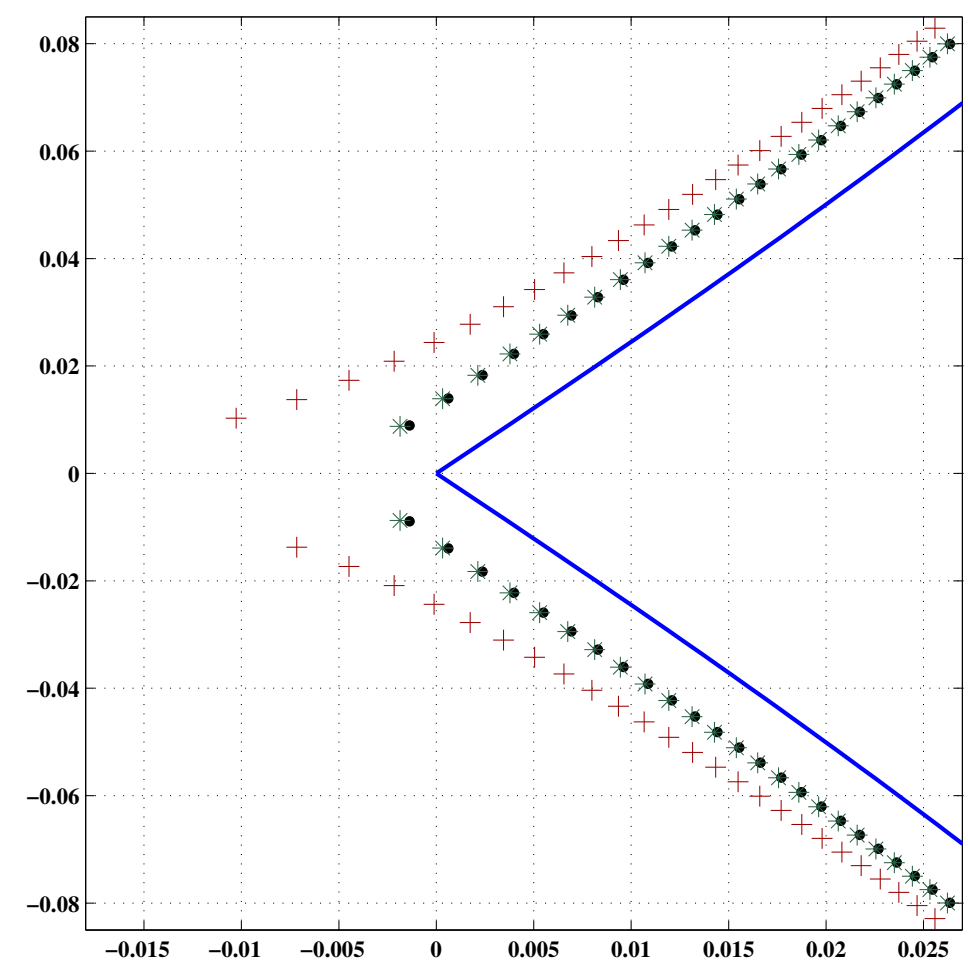

FiguRE 4 . The picture shows a piece of $\mathcal{R}(a)$ for the symbol $a(t)=t^{-1}(1-t)^{3 / 4}$ (solid line), located "close" to zero. The dots are sp $T_{4096}(a)$ calculated by Matlab. The crosses and the stars are the approximations obtained by using 2 and 3 terms of (1.5), respectively. 


\section{References}

[1] E. Basor and K. E. Morrison, The Fisher-Hartwig conjecture and Toeplitz eigenvalues. Linear Alg. Appl. 202 (1993), 129-142.

[2] G. Baxter and P. Schmidt, Determinants of a certain class of non-Hermitian Toeplitz matrices. Math. Scand. 9 (1961), 122-128.

[3] J. M. Bogoya, Toward the limiting set of a Toeplitz operator with non-rational symbol. Master thesis, CINVESTAV del I.P.N., Ciudad de México, 2008.

[4] A. Böttcher and S. Grudsky, Asymptotic spectra of dense Toeplitz matrices are unstable. Numerical Algorithms 33 (2003), 105-112.

[5] A. Böttcher and S. M. Grudsky, Spectral Properties of Banded Toeplitz Matrices. SIAM, Philadelphia, 2005.

[6] A. Böttcher, S. M. Grudsky, and E. A. Maksimenko, Inside the eigenvalues of certain Hermitian Toeplitz band matrices. J. Comput. Appl. Math. 233 (2010), 2245-2264.

[7] A. Böttcher, S. M. Grudsky, E. A. Maksimenko, and J. Unterberger, The first order asymptotics of the extreme eigenvectors of certain Hermitian Toeplitz matrices. Integral Equations Operator Theory 63 (2009), 165-180.

[8] A. Böttcher and B. Silbermann, Introduction to Large Truncated Toeplitz Matrices. Universitext, Springer-Verlag, New York, 1999.

[9] A. Böttcher and B. Silbermann, Analysis of Toeplitz Operators. 2nd edition, Springer-Verlag, Berlin, Heidelberg, New York, 2006.

[10] J. B. Conway, A Course in Functional Analysis. Springer-Verlag, New York, 1985.

[11] H. Dai, Z. Geary, and L. P. Kadanoff, Asymptotics of eigenvalues and eigenvectors of Toeplitz matrices. J. Stat. Mech. Theory Exp., P05012 (2009), 25 pp.

[12] K. M. Day, Measures associated with Toeplitz matrices generated by the Laurent expansion of rational functions. Trans. Amer. Math. Soc. 209 (1975), 175-183.

[13] U. Grenander and G. Szegö, Toeplitz Forms and Their Applications. University of California Press, Berkeley and Los Angeles, 1958.

[14] M. V. Fedoryuk, The Saddle-Point Method. Nauka, Moscow, 1977 [Russian].

[15] I. I. Hirschman, Jr., The spectra of certain Toeplitz matrices. Illinois J. Math. 11 (1967), 145-159.

[16] M. Kac, W. L. Murdock, and G. Szegö, On the eigenvalues of certain Hermitian forms. J. Rational Mech. Anal. 2 (1953), 767-800.

[17] S.-Y. Lee, H. Dai, and E. Bettelheim, Asymptotic eigenvalue distribution of large Toeplitz matrices. arXiv:0708.3124v1.

[18] A. Yu. Novosel'tsev and I. B. Simonenko, Dependence of the asymptotics of extreme eigenvalues of truncated Toeplitz matrices on the rate of attaining the extremum by the symbol. St. Petersburg Math. J. 16 (2005), 713-718.

[19] P. Schmidt and F. Spitzer, The Toeplitz matrices of an arbitrary Laurent polynomial. Math. Scand. 8 (1960), 15-38.

[20] S. Serra Capizzano, On the extreme spectral properties of Toeplitz matrices generated by $L^{1}$ functions with several minima/maxima. BIT 36 (1996), 135-142.

[21] S. Serra Capizzano, On the extreme eigenvalues of Hermitian (block) Toeplitz matrices. Linear Algebra Appl. 270 (1998), 109-129. 
[22] S. Serra Capizzano and P. Tilli, Extreme singular values and eigenvalues of nonHermitian block Toeplitz matrices. J. Comput. Appl. Math. 108 (1999), 113-130.

[23] S. V. Parter, Extreme eigenvalues of Toeplitz forms and applications to elliptic difference equations. Trans. Amer. Math. Soc. 99 (1961), 153-192.

[24] S. V. Parter, On the extreme eigenvalues of Toeplitz matrices. Trans. Amer. Math. Soc. 100 (1961), 263-276.

[25] P. Tilli, Some results on complex Toeplitz eigenvalues. J. Math. Anal. Appl. 239 (1999), 390-401.

[26] E. E. Tyrtyshnikov and N. L. Zamarashkin, Spectra of multilevel Toeplitz matrices: advanced theory via simple matrix relationships. Linear Alg. Appl. 270 (1998), 15-27.

[27] H. Widom, On the eigenvalues of certain Hermitian operators. Trans. Amer. Math. Soc. 88 (1958), 491-522.

[28] H. Widom, Eigenvalue distribution of nonselfadjoint Toeplitz matrices and the asymptotics of Toeplitz determinants in the case of nonvanishing index. Oper. Theory: Adv. Appl. 48 (1990), 387-421.

[29] N. L. Zamarashkin and E. E. Tyrtyshnikov, Distribution of the eigenvalues and singular numbers of Toeplitz matrices under weakened requirements on the generating function. Sb. Math. 188 (1997), 1191-1201.

[30] P. Zizler, R. A. Zuidwijk, K. F. Taylor, and S. Arimoto, A finer aspect of eigenvalue distribution of selfadjoint band Toeplitz matrices. SIAM J. Matrix Anal. Appl. 24 (2002), 59-67.

J. M. Bogoya

CINVESTAV del I.P.N.

Departamento de Matemáticas

Apartado Postal 14-740

07000 Ciudad de México

México

e-mail: mbogoya@math.cinvestav.mx

A. Böttcher

Technische Universität Chemnitz

Fakultät für Mathematik

09107 Chemnitz

Germany

e-mail: aboettch@mathematik.tu-chemnitz.de

S. M. Grudsky

CINVESTAV del I.P.N.

Departamento de Matemáticas

Apartado Postal 14-740

07000 Ciudad de México

México

e-mail: grudsky@math.cinvestav.mx 